\title{
Muon Identification with the ATLAS Tile Calorimeter Read-Out Driver for Level-2 Trigger Purposes
}

\author{
A. Ruiz-Martínez on behalf of the ATLAS Collaboration
}

\begin{abstract}
The Hadronic Tile Calorimeter (TileCal) at the ATLAS experiment is a detector made out of iron as passive medium and plastic scintillating tiles as active medium. The light produced by the particles is converted to electrical signals which are digitized in the front-end electronics and sent to the backend system. The main element of the back-end electronics are the VME 9U Read-Out Driver (ROD) boards, responsible for data management, processing and transmission. A total of 32 ROD boards, placed in the data acquisition chain between the Level-1 and Level-2 trigger, are needed to read out the whole calorimeter. They are equipped with fixed-point Digital Signal Processors (DSPs) that apply online algorithms on the incoming raw data.
\end{abstract}

Although the main purpose of TileCal is to measure the energy and direction of the hadronic jets, taking advantage of its projective segmentation soft muons not triggered at Level1 (with $p_{\mathrm{T}}<5 \mathrm{GeV}$ ) can be recovered. A TileCal stand-alone muon identification algorithm is presented and its usage at Level2 could improve the overall ATLAS muon trigger in the low- $p_{\mathrm{T}}$ range. Furthermore, the implementation of this algorithm at the 8 ROD DSP level allows fast full scans on the calorimeter to trigger ó muons at Level-2, fulfilling the latency requirements at this stage.

This paper describes the implementation of this muon identification algorithm in the ROD DSPs and its performance on Monte Carlo data as well as the results on real data obtained

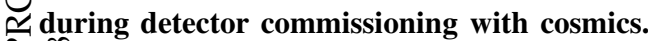

,

TLAS [1] is a general-purpose experiment for the Large Hadron Collider (LHC) [2] at CERN, whose first beams were circulated on September 10th 2008, and first collisions are foreseen for 2009. Although the main goal of ATLAS is zen discovery of the Higgs boson or New Physics beyond the Standard Model, it represents an extraordinary opportunity for B-Physics studies [3], where the muon trigger algorithm presented here will be used. Fig. 1 shows the ATLAS experiment and its subdetectors.

The ATLAS Hadronic Tile Calorimeter (TileCal) [4] is a sampling device formed by iron as passive material and plastic scintillating plates placed in perpendicular direction to the beam pipe as active medium. It is divided in a central barrel $(|\eta|<1.0)$ and two extended barrels $(0.8<|\eta|<1.7)$, with each barrel formed by 64 modules in the $\phi$ direction. The modules are divided in cells, which are grouped radially in three layers (named A, BC and D from the innermost to the

A. Ruiz-Martínez (e-mail: aranzazu@mail.cern.ch) is with the Departamento de Física Atómica, Molecular y Nuclear and IFIC, CSIC-Universitat de València, Spain.

Manuscript received November 14, 2008. outermost layer) and with an $\eta$-projective geometry, as Fig. 2 shows. Each cell is read out by two photomultiplier tubes (PMTs) and each PMT corresponds to one read-out channel.

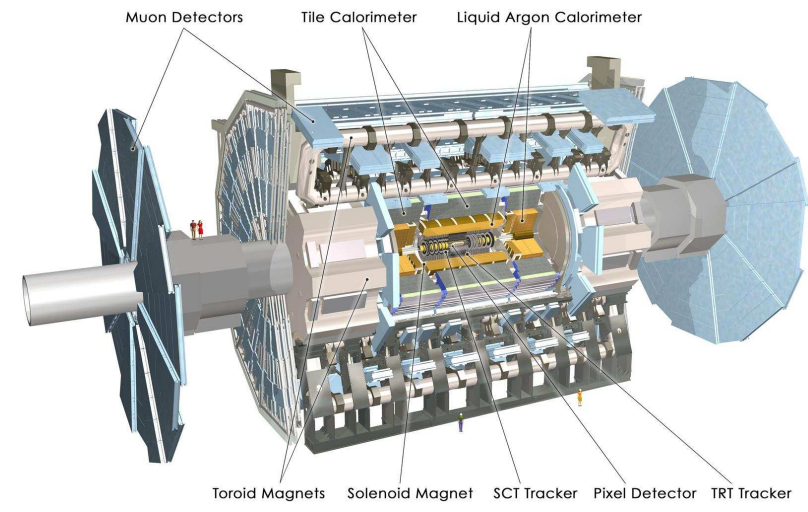

Fig. 1. Drawing of the ATLAS experiment showing all its subdetectors.

The energy deposited by the particles going through the tiles produces light which is conducted by wavelength shifting fibers to the front-end electronics located in the outermost part of the modules. The light is converted to electrical signals which are digitized each $25 \mathrm{~ns}$. These digital samples are sent from the front-end to the back-end system with optical fibers.
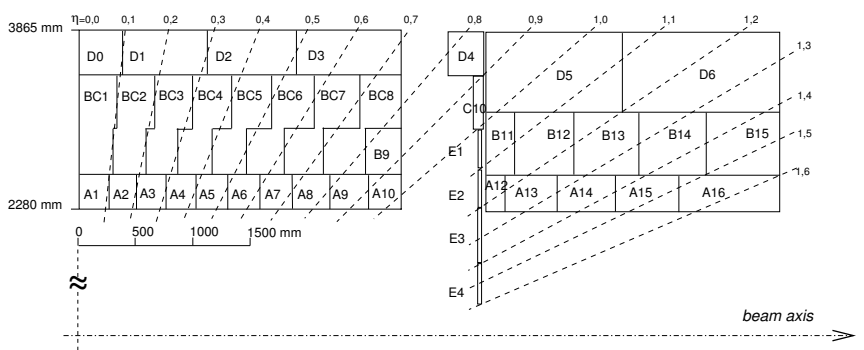

Fig. 2. Diagram of the TileCal cell segmentation. Note the three longitudinal layers and the $\eta$-projective towers.

The main element of the back-end electronics is the ReadOut Driver (ROD) [5]. It is a 9U VME board (see Fig. 3) responsible of data format, management and transmission at a maximum Level-1 trigger rate of $100 \mathrm{kHz}$. A total of $32 \mathrm{ROD}$ modules are needed to read out the whole calorimeter. Data are buffered on the detector while the Level-1 trigger makes its selection, and only the data corresponding to positive Level-1 triggers are sent to the RODs. 
The ROD boards contain two processing unit daughterboards equipped with TMS320C6414 $\mathrm{x}^{\mathrm{TM}}$ fixed-point Digital Signal Processors (DSPs) to process the incoming raw data. The energy and timing of the signals are reconstructed using Optimal Filtering [6] for each channel and sent to the next step in the data acquisition chain. The capabilities of the DSP allow it to apply trigger-oriented algorithms in real time over the incoming data. A TileCal muon identification (TileMuId) [7] algorithm has been implemented in this device to be used at the Level-2 trigger.

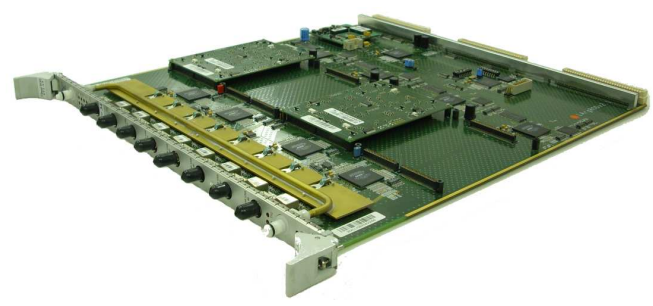

Fig. 3. Picture of the Read-Out Driver motherboard with the two DSP processing unit mezzanine boards.

\section{Algorithm Description}

Muons with low transverse momentum $\left(p_{\mathrm{T}}\right)$ are the characteristic signature for several B-physics processes. The ATLAS muon spectrometer efficiency to trigger muons with $p_{\mathrm{T}}<5 \mathrm{GeV}$ decreases significantly [8] due to the bending of the muon tracks by the magnetic field. For instance, very low- $p_{\mathrm{T}}$ muons may not reach the middle station of the barrel muon spectrometer. Some of these muons can be recovered using information from TileCal at Level-2 trigger.

An algorithm to identify low $p_{\mathrm{T}}$ muons, TileMuId, based on the energy deposited in cells with an $\eta$-projective pattern has been implemented in the TileCal ROD DSP core. This algorithm takes advantage, on one hand, of the typical energy deposition of the muons and, on the other hand, of the geometrical segmentation of the calorimeter.

The muon search in TileMuId starts from the outermost layer looking for any energy deposition in a single cell compatible with a minimum ionizing particle (MIP), defining the first candidates to muons. The condition required is that the energy deposition in the cell is comprised between a lower and a higher energy thresholds:

$$
\mathrm{Thr}_{\text {lower }} \leq E_{\text {cell }} \leq \mathrm{Thr}_{\text {higher }}
$$

If the candidate found in the D layer is confirmed by cells in the BC and A layers following a projective pattern in $\eta$ and having energies also compatible with a MIP (as Fig. 4 shows), the muon is tagged.

In addition, two strategies have been developed:

- Tight selection: MIP-like energy deposition is required in the three layers.

- Loose selection: In order to be efficient on events in which the muon loses a considerable fraction of its energy in one of the layers, muons are also tagged if the energy deposition in one layer exceeds the higher threshold.
The set of thresholds used is determined and optimized for each cell depending on its size and $\eta$ coordinate. The lower energy threshold is meant to cut the electronic noise and the minimum bias pileup events. The higher energy threshold is used to eliminate the hadronic showers.

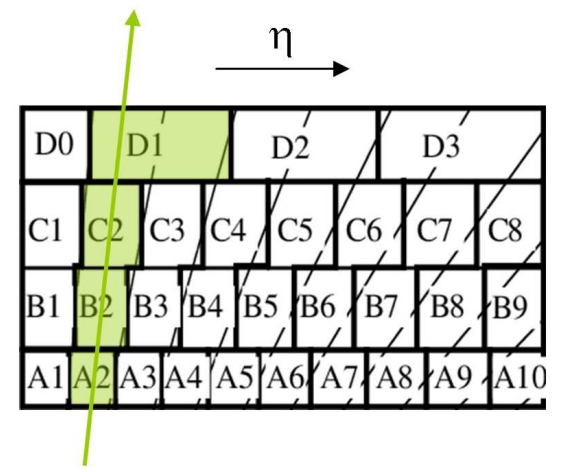

Fig. 4. Diagram of a muon going through TileCal, with the cells used by the TileMuId algorithm for its identification highlighted.

This algorithm is meant to be used at the Level-2 trigger, for the selection of B-Physics channels which requires soft muons in the final state. Currently, there are two trigger implementations in Athena (general framework for the ATLAS offline software) being used:

- TileLookForMu: Level-2 trigger algorithm fully implemented inside the Athena framework.

- TileRODMu: Level-2 trigger algorithm implemented in Athena that collects the muons found previously at the ROD level. This way, TileMuId, once implemented in the ROD DSP core, is processed in parallel over all the modules and its output is encoded and sent in the ROD data format to the Level-2 trigger.

With its implementation in the ROD DSPs, the information from TileMuId is inserted in the data stream as a ROD subfragment, which can be accessed at Level-2 trigger with low consumption of processing time. This way the information about low- $p_{\mathrm{T}}$ muons in TileCal is sent for all the events accepted by Level-1 trigger and by means of full scans at Level-2 trigger, new Regions of Interest can be created.

\section{Algorithm Performance}

The performance of the ROD-based version of the algorithm (TileRODMu) has been studied in terms of efficiency and fraction of fakes with Monte Carlo data and the results obtained have been compared with the fully offline implementation of the TileMuId algorithm (TileLookForMu).

In addition, the results obtained executing the algorithm online in the ROD DSPs are shown with real data from the ATLAS commissioning with cosmic rays.

\section{A. Performance with Monte Carlo Data}

In order to distinguish whether a tagged muon corresponds to an actual muon in the simulation an acceptance cone of $\Delta \eta \times \Delta \phi=0.2 \times 0.12$ is used. The dimensions of the cone are defined with single muon events as $4 \sigma$ of the distributions 
of the residuals. These distributions are shown in Fig. 5 and 6 for the case of TileRODMu, containing the difference between the $\eta$ and $\phi$ coordinates of the muons tagged by the algorithm and the coordinates of the muon in the Monte Carlo truth information. Note that the dimensions of the cone in $\eta$ and $\phi$ are comparable with the cell size in the D layer.

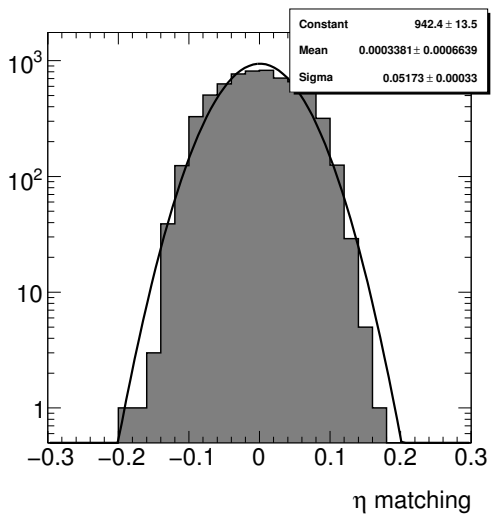

Fig. 5. Distribution of residuals in $\eta$ for TileRODMu. The distribution is fitted with a Gaussian.

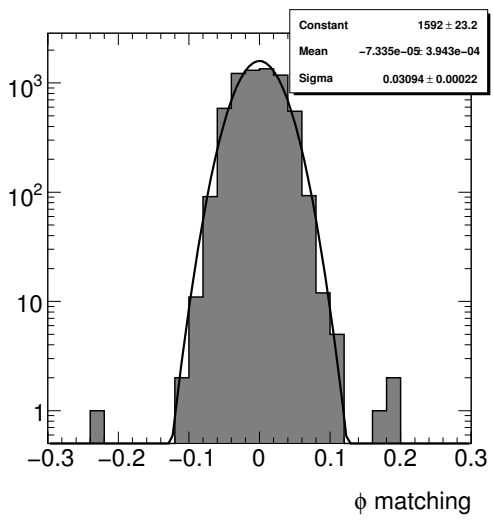

Fig. 6. Distribution of residuals in $\phi$ for TileRODMu. The distribution is fitted with a Gaussian.

Efficiency and fraction of fakes are evaluated using the inclusive $\mathrm{b} \overline{\mathrm{b}} \rightarrow \mu(6) \mathrm{X}$ Monte Carlo process, with at least one muon with $p_{\mathrm{T}}>6 \mathrm{GeV}$ in the final state. This sample is commonly used for trigger studies at Level-2 since the events with a muon with $p_{\mathrm{T}}>6 \mathrm{GeV}$ are accepted by Level-1 inclusive muon triggers with high efficiency.

The efficiency is computed as:

$$
\text { Efficiency }=\frac{\text { Number of tagged muons }}{\text { Number of generated muons }} \text {. }
$$

Only muons generated with $p_{\mathrm{T}}>2 \mathrm{GeV}$ in $|\eta|<1.4$ (coverage of the TileCal third layer) have been considered.

The efficiency as a function of $\eta$ (Fig. 7) presents different structures. The central region $(|\eta|<0.8)$ corresponding to the TileCal long barrel modules presents a flat efficiency with values above $80 \%$, except for $|\eta| \sim 0$ where the calorimeter sampling ratio is less uniform. In the gap between long and extended barrels TileRODMu cannot be applied due to the fact that these data are processed by different RODs. Due to the fact that TileCal is symmetric in $\phi$, the efficiency is uniform as a function of this coordinate as Fig. 8 shows.

The efficiency of the TileRODMu algorithm as a function of the muon $p_{\mathrm{T}}$ (Fig. 9) is flat for muons with $p_{\mathrm{T}}>3 \mathrm{GeV}$, allowing a good performance in the low- $p_{\mathrm{T}}$ range which indeed complements the performance of the ATLAS muon spectrometer.

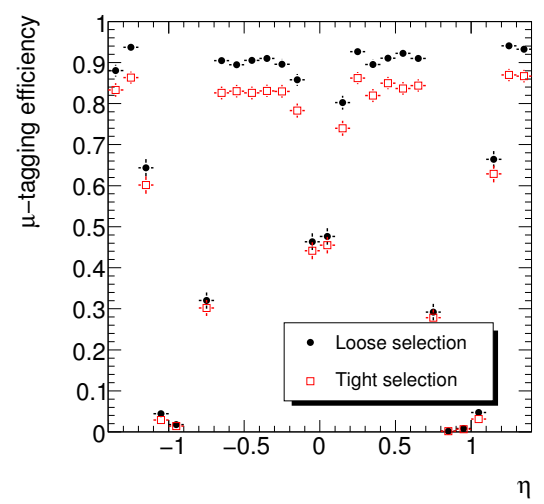

Fig. 7. Efficiency as a function of $\eta$ for the loose selection (filled circles) and tight selection (open squares) using TileRODMu.

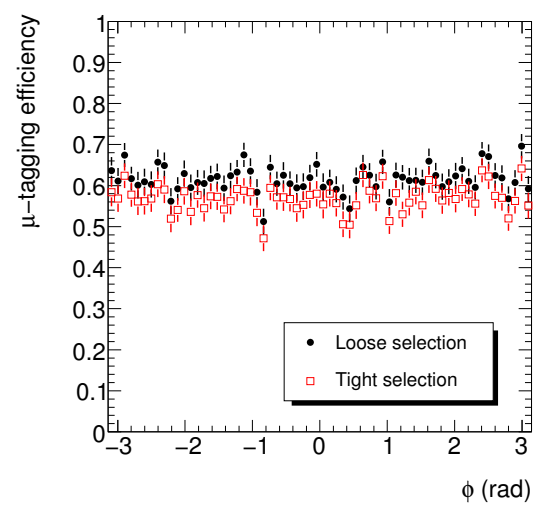

Fig. 8. Efficiency as a function of $\phi$ for the loose selection (filled circles) and tight selection (open squares) using TileRODMu.

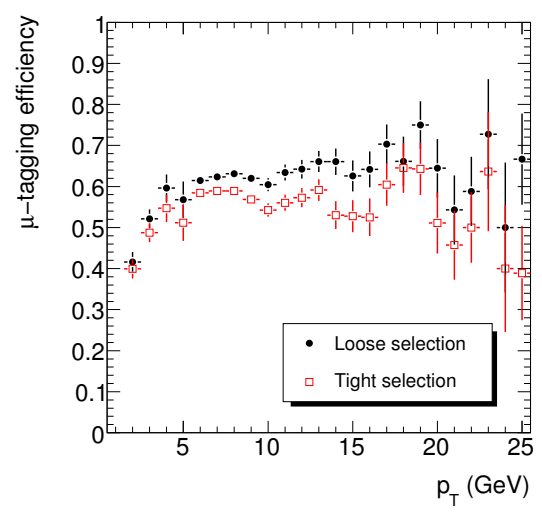

Fig. 9. Efficiency as a function of $p_{\mathrm{T}}$ for the loose selection (filled circles) and tight selection (open squares) using TileRODMu. 
The fraction of fakes (mistagged muons) given by the algorithm has been defined as:

$$
\text { Fraction of fakes }=\frac{\text { Number of mistagged muons }}{\text { Number of events }} .
$$

The fraction of fakes as a function of $\eta$ and $\phi$ (normalized to the total number of events) are shown in Fig. 10 and 11, respectively. Again a flat distribution in $\phi$ is found but most of the fakes appear at high- $\eta$ values, that is, in the extended barrel where cells are bigger and the projectivity is worse.

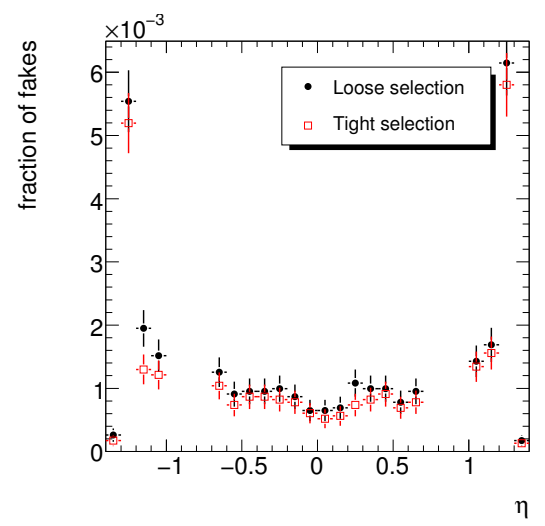

Fig. 10. Fraction of fakes as a function of $\eta$ for the loose selection (filled circles) and tight selection (open squares) using TileRODMu.

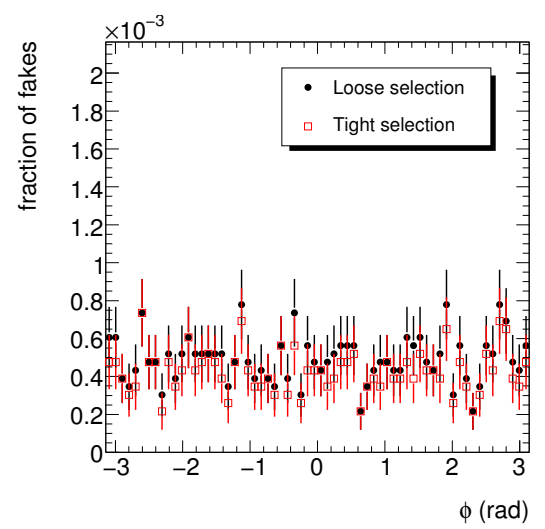

Fig. 11. Fraction of fakes as a function of $\phi$ for the loose selection (filled circles) and tight selection (open squares) using TileRODMu.

Table I summarizes the efficiencies and fraction of fakes for the two TileMuId implementations and muon selection criteria.

TABLE I

EFFICIENCY AND FRACTION OF FAKES FOR TILEMUID

\begin{tabular}{|c|c|c|}
\cline { 2 - 3 } \multicolumn{1}{c|}{} & Tight selection & Loose selection \\
\cline { 2 - 3 } \multicolumn{1}{c|}{} & \multicolumn{2}{c|}{ Efficiency (\%) } \\
\hline TileLookForMu & $71.8 \pm 0.4$ & $82.5 \pm 0.3$ \\
\hline TileRODMu & $56.9 \pm 0.4$ & $61.6 \pm 0.4$ \\
\hline & \multicolumn{2}{|c|}{ Fraction of fakes $(\%)$} \\
\hline TileLookForMu & $4.08 \pm 0.14$ & $5.96 \pm 0.17$ \\
\hline TileRODMu & $2.74 \pm 0.11$ & $3.14 \pm 0.12$ \\
\hline
\end{tabular}

In particular, for the tight selection, the efficiencies are slightly bigger for TileLookForMu $(\sim 72 \%)$ than for
TileRODMu $(\sim 57 \%)$ in average, mainly because the latter cannot be used in the gap region. The fraction of fakes is at the level of $\sim 4.1 \%$ for TileLookForMu and $\sim 2.7 \%$ for TileRODMu. As expected, the $\mu$-tagging efficiency increases when applying the loose selection criteria, where the muons which lose a considerable fraction of the energy in a single layer are also tagged, but the fraction of fakes increases as well.

\section{B. Performance with Cosmics Data}

During ATLAS commissioning, a program of cosmic rays data acquisition has been planned for TileCal stand-alone and in combination with other subdetectors. The implementation of the algorithm in the ROD DSPs has been tested with real data during the detector commissioning with the acquisition of cosmic rays.

TileMuId is meant to identify $\eta$-projective muons (generated at the interaction point). Hence, cosmics are not the ideal data source for testing the algorithm. However, these data are a great chance to study the behavior of the algorithm using real muons and in the actual TileCal noise environment during ATLAS operation. Fig. 12 shows the display of a cosmic event acquired by the ATLAS experiment using all the subdetectors in a combined way.

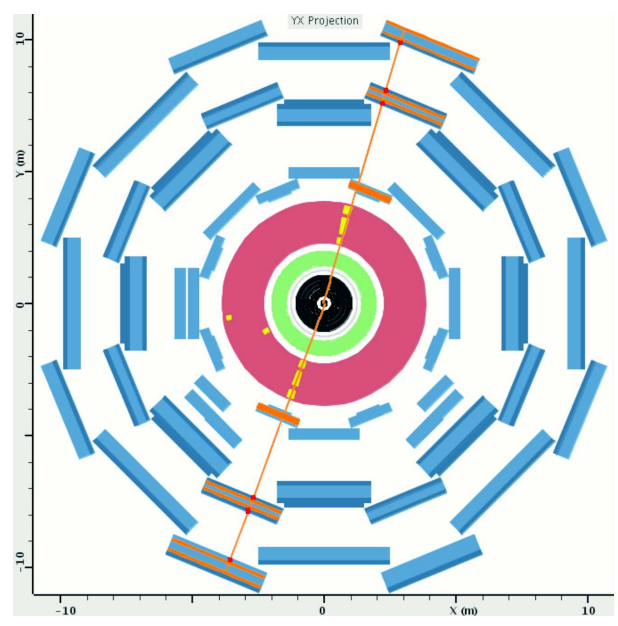

Fig. 12. Display of a cosmic event in ATLAS.

Fig. 13 shows the energy deposited in TileCal by the muons identified online in the ROD DSPs for the cosmics run 91060. Only events triggered by the Level-1 calorimeter trigger [9] have been considered. As expected, a Landau-like distribution is obtained with maximum at $\sim 2 \mathrm{GeV}$.

Fig. 14 shows the $\phi$ distribution for the tagged muons which in this case is not flat but with an excess in the top-bottom regions due to the cosmics topology.

Finally, Fig. 15 shows the correlation between the $\eta$ and $\phi$ coordinates of the muons identified with TileRODMu. The structure in $\eta$ is due to the gap between the central and the extended barrels. The empty regions at certain $\phi$ values are due to Tilecal modules which were not powered in this particular run. 


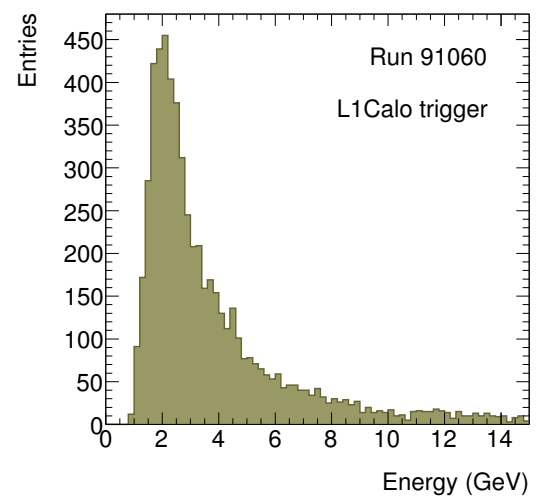

Fig. 13. Energy deposited in TileCal by the cosmic muons tagged by the ROD DSPs for run 91060.

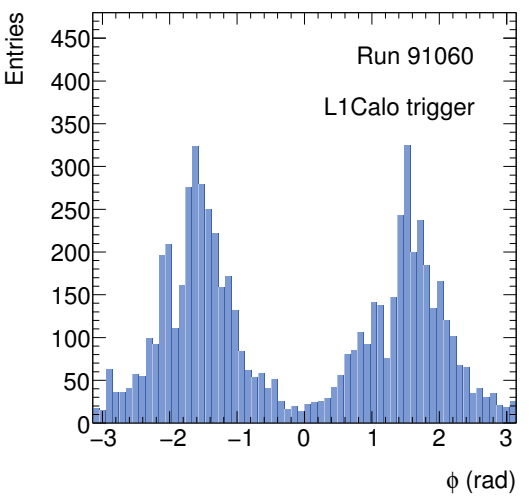

Fig. 14. Cosmic muons tagged by the ROD DSPs as a function of $\phi$ (the binning corresponds to a TileCal module, $2 \pi / 64=0.098 \mathrm{rad}$ ).

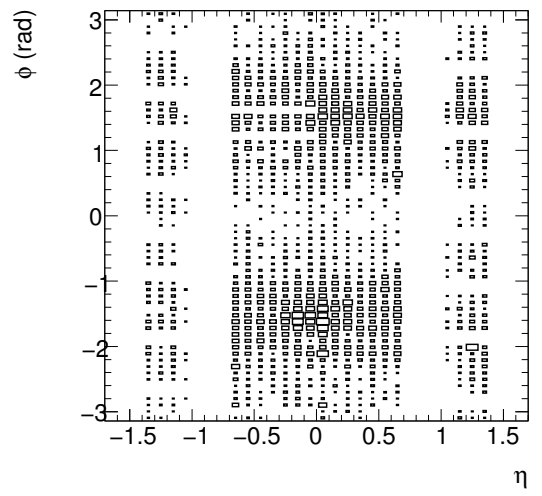

Fig. 15. Cosmic muons tagged by the ROD DSPs in the $(\eta, \phi)$ plane.

\section{CONCLusion}

This paper summarizes the implementation of a TileCal stand-alone low- $p_{\mathrm{T}}$ muon tagging algorithm inside the ROD DSPs, which can be used to enhance the performance of the ATLAS Level-2 trigger. From Monte Carlo data, high efficiencies are obtained for the algorithm $\left(\sim 60 \%\right.$ for $\left.p_{\mathrm{T}}>2 \mathrm{GeV}\right)$ in correlation with a small fraction of fakes $(\sim 2.7 \%)$. This algorithm is implemented and being used during the ATLAS commissioning where it is used to tag cosmic muons.

\section{REFERENCES}

[1] The ATLAS Collaboration, G. Aad et al., "The ATLAS Experiment at the CERN Large Hadron Collider", JINST 3, S08003 (2008).

[2] L. Evans and P. Bryant, "LHC Machine", JINST 3, S08001 (2008).

[3] The ATLAS Collaboration, "ATLAS detector and physics performance: Technical Design Report, 2", CERN-LHCC-99-015, 1999.

[4] The ATLAS Collaboration, "ATLAS tile calorimeter: Technical Design Report", CERN-LHCC-96-042, 1996.

[5] J. Castelo et al., "TileCal ROD Hardware and Software Requirements", ATLAS Note CERN-ATL-TILECAL-2005-003, 2005.

[6] B. Salvachúa et al., "Algorithms for the ROD DSP of the ATLAS Hadronic Tile Calorimeter", JINST 2, T02001 (2007).

[7] G. Usai, "Trigger of low $p_{\mathrm{T}}$ muons with the ATLAS hadronic calorimeter", Nucl. Instrum. Meth. A 518, 36 (2004).

[8] G. Aielli et al., "The RPC first level muon trigger in the barrel of the ATLAS experiment", Nucl. Phys. Proc. Suppl. 158, 11 (2006).

[9] R. Achenbach et al., "The ATLAS level-1 calorimeter trigger", JINST 3, P03001 (2008) 